\title{
Repercussões Maternas e Perinatais Relacionadas à Classificação da Hipertensão Arterial na Gravidez
}

Autor: Milton Marchioli

Orientador: Prof. Dr. José Carlos Peraçoli

Tese de Mestrado apresentada ao Departamento de Ginecologia e Obstetrícia da Faculdade de Medicina de Botucatu - UNESP em 7/5/99.

A hipertensão arterial representa importante fator de morbimortalidade materna e fetal. A forma como se manifesta na gestação parece ter forte impacto no resultado materno e perinatal. Este trabalho teve como objetivo analisar a influência da hipertensão arterial sobre a mãe e o produto da concepção, estimando dentro de e comparando entre cada tipo de hipertensão arterial, a incidência de: intercorrências obstétricas, cesárea, mortalidade materna, peso do recém-nascido e sua classificação relacionada à idade gestacional, óbito fetal e neonatal e taxa de mortalidade perinatal. Por meio de estudo retrospectivo, foram analisados 530 casos de hipertensão arterial na gravidez, divididos em cinco grupos, de acordo com o tipo de hipertensão arterial: hipertensão arterial crônica (HAC), hipertensão induzida pela gestação (HIG), pré-eclâmpsia (PE), hipertensão arterial crônica superposta a hipertensão induzida pela gestação (HAC + HIG) e hipertensão arterial crônica superposta a pré-eclâmpsia (HAC $+\mathrm{PE})$. A HAC isolada e superposta a HIG ou PE $(42,8 \%)$ e a HIG isolada $(39,3 \%)$ ocorreram com maior freqüência. As gestantes portadoras de HAC isolada ou superposta por HIG ou PE eram mais idosas e tinham maior número de gestações, enquanto as portadoras de HIG ou PE eram na maioria primigestas. Em todos os grupos o antecedente familiar de hipertensão arterial esteve presente em mais que $50 \%$ dos casos. Os casos de PE isolada e de HAC superposta a HIG ou PE foram considerados os mais graves, quando se analisou tanto o valor da pressão arterial, sistólica e diastólica, como o quadro hipertensivo como um todo. A PE isolada foi o grupo com maior incidência de intercorrências obstétricas, dentre as quais predominou a eclâmpsia iminente $(20,9 \%)$. A taxa de cesárea foi elevada em todos os grupos $(75,2 \%)$, principalmente na PE $(87,2 \%)$. Os resultados perinatais mostraram maior incidência de fetos pré-termo, com peso pequeno para idade gestacional e peso médio menor que 2.500 gramas, na $\mathrm{PE}$ isolada e HAC superposta a HIG ou PE. A mortalidade perinatal foi maior na $\mathrm{HAC}+\mathrm{PE}$ e a materna na PE. Os resultados obtidos permitem concluir que, em gestantes hipertensas, é importante identificar o tipo de hipertensão arterial, uma vez que na $\mathrm{PE}$ isolada e na HAC superposta a HIG ou PE, encontramos maior incidência de casos graves e complicações obstétricas, assim como os piores resultados maternos e fetais. Portanto, nesses tipos de hipertensão arterial, há necessidade de controle mais rigido, para melhorar o prognóstico materno e fetal.

Palavras-chave: Gravidez. Hipertensão arterial. Mortalidade materna. Mortalidade perinatal.

Avaliação da Qualidade da Assistência às Gestações Pós-Termo nas Maternidades Públicas do Rio de Janeiro. Utilização de Procedimentos de Indução

Aluno: Neiw Oliveira Iamada

Orientadores: Profa. Dr ${ }^{a}$. Letícia Krauss Silva e Prof. Dr. Aldo Franklin Reis

Dissertação de Mestrado na área de concentração Clínica Obstétrica da Universidade Federal do Rio de Janeiro, apresentada à Maternidade Escola da UFRJ em 27/5/99.

O objetivo do presente trabalho foi avaliar a qualidade da assistência às gestações pós-termo nas maternidades públicas do Rio de Janeiro, focalizando a utilização de procedimentos de indução. Os indicadores e padrões de processo e de resultados foram derivados de evidências científicas (ensaios clínicos). Para a análise de processo, o padrão derivado para a utilização da indução em uma conduta eletiva ao final da $42^{\mathrm{a}}$ semana foi de $100 \%$. Para a análise de resultados, os indicadores utilizados foram a mortalidade perinatal e a taxa de cesariana. Foram também construídos outros padrões de processo, aplicados ao sistema de saúde (curvas de distribuição "natural" dos nascimentos e estrutura de dilatação à admissão). O uso da tecnologia de indução pelas maternidades públicas do Rio de Janeiro, no período 1988-90, em gestantes pós-termo admitidas fora de trabalho de parto, foi de $18.8 \%$ (referente aos ensaios). A mortalidade perinatal pós- termo observada nas maternidades para o período em questão foi de 10.1/1000 nascimentos, excluídos os casos de malformações congênitas. A mortalidade perinatal das pacientes pós-termo com complicações maternas e obstétricas foi de 32/1000 nascimentos; para o conjunto total de pacientes pós-termo, excluindo malformações, foi de 15.2/1000 nascimentos. A comparação da curva correspondente à estrutura da idade gestacional ao parto das pacientes pós-termo das maternidades públicas do Rio de Janeiro com as referidas curvas de "não-intervenção" mostrou serem as mesmas similares. Conclusões: Os achados do estudo foram consistentes em demonstrar, nos serviços estudados, uma baixa efetividade na assistência pública (pré-natal e maternidades) às gestações pós-termo.

Palavras chaves: Gravidez normal, Trabalho de parto, Resolução da gravidez, Assistência ao parto. 